\title{
Cross-Cultural Differences in Map Design Perception
}

\author{
Bibigul Zhunis ${ }^{\mathrm{a}}$ *, Georg Gartner ${ }^{\mathrm{b}}$, Silvia Klettner ${ }^{\mathrm{b}}$ \\ ${ }^{a}$ GIS Analyst, TU Wien, Austria - bibishkazh@gmail.com \\ ${ }^{b}$ TUWien, Austria - georg.gartner@tuwien.ac.at; silvia.klettner@tuwien.ac.at \\ * Corresponding author
}

Keywords: topographic map design, cognitive development, cross-cultural differences in cognitive styles, cultural dimensions.

\section{Abstract:}

It is common knowledge that the visual appearance of a map makes a significant contribution to its quality and functionality. But do we perceive it differently at the scale of cultures? Are there any group behavioural patterns in cognitive performance whilst communicating a map? This research aims to investigate to what extent a cultural background influences the process of interaction (perception, attention, learning, and interpretation of the cartographic information) with a specific topographic map design. Particularly, it was examined through the topographic map variables due to its exceptional design styles specific to one country.

An interesting comparison was illustrated in Norenzayan et al.'s work (2007) where the human mind and cognition were analogous to the computer machine. In line with it, the input differs across cultures, due to ecological, social conditions, and the output is in the form of beliefs and behaviour. Similarly, Segall (1963) posited that the input expresses "perceptual inference habits", Bender and Beller (2011) have concluded that cognition is a result of processing and content. While the processing part is universal to all human beings and separate from the context (i.e. cultural background), the content differs across cultures. Bengston (as cited in Holland, 1982) stated that cognitive style is individual difference variables that presumably describe a person's preferred and characteristic way of perceiving, learning, and thinking. Bearing in mind that map users may have different cognitive styles and abilities, Montello (2004), Oyserman (2011) stressed the role of culture (i.e., situated cognition).

With this in mind, this paper aimed to address the following research questions: Do people perceive and process cartographic information differently? Is there a presence of cross-cultural background in a "map-user" interaction? If yes, how and to what extent does it influence cognitive abilities and style? To answer these questions, a user study on the detection of differences in cognitive performance through map-reading tasks and topographic map design assessment was performed. It was executed among participants from European and Central Asian countries who were assumed to have different cultural backgrounds and mindsets.

The map samples were created replicating the topographic map design style of two national mapping agencies - Federal Office of Metrology and Surveying of the Federal Ministry of Digital and Economic Affairs of the Republic of Austria (Map I) and Committee of Geodesy and Cartography of the Ministry of Digital Development, Innovation and Aerospace Industry of the Republic of Kazakhstan (Map II). The user-study materials were created for experimental purposes, and it was essential to avoid any familiarity. Having in mind that the aspects of visual balance and simplicitycomplexity play a significant role in that case, the level of map elements' density on two map samples was brought to one level so that some map elements were reduced.

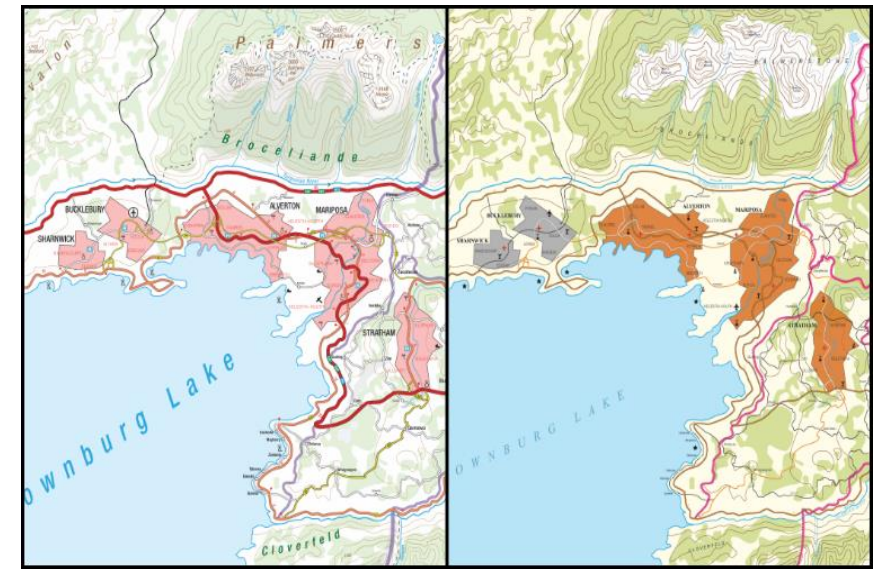

Figure 1. Map samples - Map I (“Austrian style”) and Map II ("Kazakhstani style")

In the study interviews with 50 participants aged between 15 and 40 were carried out. The interviewees were divided into two groups (i.e. Western - Group 1 and Central Asian - Group 2) based on their background determined by cultural, environmental dimensions, and psychological aspects. The first group consisted of participants from the European countries, including Germany $(n=6)$, Bosnia and Herzegovina $(n=2)$, Russian Federation $(n=2)$, Great Britain $(n=2)$ while the second was represented by map users from Central Asian countries - Kazakhstan $(\mathrm{n}=22)$ and Kyrgyzstan $(\mathrm{n}$ $=3$ ).

"Thinking aloud" interviews were carried out online due to the diverse location of the participants and current pandemic situation constraints. It additionally allowed to give oral instructions, measure directly the time spent on each task, observe 
the behaviour, and overall supervise the process. A hybrid (both qualitative and quantitative) approach was applied to focus on current research questions. Quantitative measurements were intended to examine the state of cognitive abilities like attention, perception, and learning between two user groups by recording the time needed to solve the map-reading tasks in finding a labelled place on two map samples. In the second part of the experiment, participants were invited to assess the design/aesthetic component of two distinct map samples and subsequently share their opinion, associations, logical strategies, and feedback during the whole session. At this point, a noticeably shortened version of the AttrakDiff evaluation method (Hassenzahl, 2003; 2005) was applied and resulted in bipolar semantic differential 5-scale ranking characterizing the negative and positive variables (Ugly-Attractive, Discouragement-Motivation, Confusion-Clarity).

The main findings of the think-aloud procedure highlighted a significant difference between the given ranking values using the aforementioned qualitative evaluation method for both maps. The statistical interpretation of the results accepted an alternative hypothesis stating that there is a significant difference in given assessment values. It also verified the main theory "the more familiar to the user the map is, the higher it will be assessed". Thus, participants from Group 1 (Western) ranked Map I (Austrian) higher than Map II. Taken as a whole, the resulting outcomes corroborate with the main hypothesis for $100 \%(\mathrm{n}=25)$ that the level of familiarity plays a crucial role in such types of ratings as evaluation of attractiveness and usability (Bornstein, as cited in Lee, 2001). Most of them affirmed that Map I was similar to the maps that they have been seeing and using although there were minor discrepancies. On the contrary, the respondents of Group 2 asserted that Map I confuses them or creates biases due to the pale colour scheme so that the attention was lost and did not capture the attention of the whole scene, the complexity of the road network provoked by several levels and categories, and to its high visual attention in overall composition caused by the thickness of lines and several colour hues. The overall performance of Group 2 supported the current hypothesis for $67 \%$.

Furthermore, participants were able to share their walkthrough impression and comments. The procedure of parsing the written narratives and looking for patterns has resulted in such categories as "colour scheme", "font-style", "font-size", "relief representation", "road network", "depiction of settlement areas", "graphical symbols". also provided additional support for understanding the reasoning part behind the assessment. Group 1 (Western) unanimously preferred Map I duplicating the design of the Austrian topographic map and Group 2 (Central Asian) - Kazakhstani map. Participants from Group 1 found similarities between the design of Map I and the maps that they have ever experienced, and also there was a significant positive correlation between given feedback on map samples' design and ranking values. As regards Group 2, over half of the participants $(n=13)$ reported that Map II looks more attractive and correct (e.g. "I don't like Map I because the colour scheme is too pale, the road network is too advanced, dense, and outstanding so that I feel discouraged to explore it whereas Map II is very fancy and visually appealing, natural features are visible and perfectly visualized, looks familiar and reminds school atlases or old geography books").

Taken together, the outcomes of the study pointed towards the idea of cross-cultural differences in map design perception. It revealed the presence of psychological phenomena, namely, mere exposure, perceptual fluency, and modified twofactor model. According to this, our experience creates and shapes our worldview. Since the input information and content acquired from the surrounding setting varies from culture to culture, our study provided insight into the theory that how we perceive visual information to some extent is influenced by cultural circumstances. Particularly, consistent assessment ranking and feedback from Group I highlighted a significant level of the importance of cultural background whilst communicating the map and fully confirmed it. Meanwhile, another group only partially (67\%) supported this theory because of the contradicting situation between rating evaluation and shared feedback on it.

This paper has presented methodological and interdisciplinary analysis in the area of cartography, cross-cultural and cognitive psychology. Considerable attention was paid to the influence of the cultural background (to which cultural environment they attach themselves), map experience level (novices, competent, and expert users) the format of experimental materials (digital), hardware characteristics (screen size and resolution, computer system properties), professional experience (familiarity or frequent use of maps in their jobs). Additionally, it has revealed promising topics for further examination, namely, the investigation of colour scheme, graphical symbols, the level of map content complexity, and visual hierarchy. However, future research needs to determine participants' cultural background more accurately, have a larger sample size taking into account the level of familiarity with maps, and provide the same experimental environment for eliminating the noise in results.

\section{References:}

Bender, A., \& Beller, S. (2011). The cultural constitution of cognition: Taking the anthropological perspective. Front. Psychology, 2(67);

Hassenzahl, M. (2003). The Thing and I: Understanding the Relationship Between User and Product. In Funology. Human-Computer Interaction Series. Blythe M.A., Overbeeke K., Monk A.F., Wright P.C. (eds) (Vol. 3). Springer, Dordrecht;

Hassenzahl, Marc. (2005). The Thing and I: Understanding the Relationship Between User and Product. In Funology: From Usability to Enjoyment (Vol. 3, pp. 31-42);

Holland, R. P. (1982). Learner Characteristics and Learner Performance: Implications for Instructional Placement Decisions. The Journal of Special Education, 16(1), 7-20; 
Lee, A. Y. (2001). The Mere Exposure Effect: An Uncertainty Reduction Explanation Revisited. Personality and Social Psychology Bulletin, 27(10), 1255-1266;

Montello, D. R. (2004). Cognition of Geographic Information. In A Research Agenda for Geographic Information Science Edited by Robert B.McMaster, E.Lynn Usery (1st ed., pp. 61-91). CRC Press;

Norenzayan, A., Choi, I., \& Peng, K. (2007). Perception and cognition. In Handbook of cultural psychology. (pp. 569594). The Guilford Press;

Oyserman, D. (2011). Culture as situated cognition: Cultural mindsets, cultural fluency, and meaning making. European Review of Social Psychology, 22(1), 164-214;

Segall, M. H., Campbell, D. T., \& Herskovits, M. J. (1963). Cultural Differences in the Perception of Geometric Illusions. Science, 139(3556), 769; 